\title{
Nurses' Professional Commitment and Its Effect on Patient Safety
}

\author{
Zaid Al-Hamdan RN, $\mathrm{PhD}^{1}$, Heyam Dalky, $\mathrm{PhD} \mathrm{RN}^{1}$ \& Jehan Al-Ramadneh, MSN, RN ${ }^{1}$ \\ ${ }^{1}$ Department of Community/Mental Health Nursing, Faculty of Nursing, Jordan University of Science \& \\ Technology, Irbid, Jordan \\ Correspondence: Zaid Al-Hamdan, Associate Professor, Faculty of Nursing, Jordan University of Science and \\ Technology, P.O.Box 3030, Irbid 22110, Jordan. Tel: 96-279-585-3482. E-mail: zaid_hamdan@hotmail.com; \\ zmhamdan@just.edu.jo
}

\author{
Received: October 28, 2017 Accepted: November 20, 2017 Online Published: December 1, 2017 \\ doi:10.5539/gjhs.v10n1p111 URL: https://doi.org/10.5539/gjhs.v10n1p111
}

\begin{abstract}
Aims and objectives: The project was designed to assess the level of professional commitment among Jordanian Registered Nurses and examine how professional commitment among nurses relates to patients' safety.

Background: Professional commitment has received a great deal of interest worldwide. Nurses constitute the largest group of healthcare professionals that spend a majority of their time at the bedside in direct patient care. Nurses have an important role in improving patient safety and providing quality of care.
\end{abstract}

Design: A descriptive, cross-sectional, correlational design was used to answer the research questions.

Methods: A convenience sampling of 180 nurses selected from three accredited hospitals (governmental, private and university-affiliated teaching hospitals) completed two questionnaires; Professional Commitment Questionnaire (PCQ) and a Patient Safety Scale. Descriptive statistics, correlation coefficient, independent sample t-test, and one-way ANOVA test were used in data analysis.

Result: Nurses' professional commitment was significantly and positively correlated with patient safety. Registered nurses perceived that their level of commitment was medium $(\mathrm{M}=3.47 ; \mathrm{SD}=1.58$ of a 7 point scale), with the highest mean recorded for nurses working in governmental hospitals $(\mathrm{M}=3.88 ; \mathrm{SD}=1.53)$. The level of perception of issues related to patient safety was slightly higher than the midpoint $(\mathrm{M}=5.94 ; \mathrm{SD}=1.38$ of a 9 point scale). Nurses' professional commitment was influenced by gender $t(158=-2.33 ; \mathrm{p}=.02)$, nursing experience in current hospitals $(r=-0.193 ; p=0.01)$, current hospital sector $(F=4.334, p=0.01)$, and monthly salary $(F=12.327$, $p=0.000)$. Patient safety was influenced by nurses' educational level $(F=3.306, p=0.03)$.

Conclusion: This study provides a preliminary understanding of how professional commitment of registered nurses can enhance patient safety. Managerial support was deemed necessary to enhance nurses' professional commitment, which, in turn, improves healthcare outcomes.

Keywords: Jordan, nurses, professional commitment, patient safety, patient-perceived care quality.

\section{Introduction}

Professional commitment has received a great deal of interest worldwide. Nurses constitute the largest group of health care professionals that spend a majority of their time at the bedside in direct patient care. Nurses have an important role in improving patient safety and providing quality of care (Mrayyan, 2012). As an important indicator for nursing care outcomes, patient safety is a high priority for health organizations (Robson, Clark, Pinnock, White, \& Baxendale, 2013). Patient safety focuses on preventing medical errors and adverse events, reducing risks, and minimizing incidences that may cause physical or emotional harm to patients (Gaal, 2010 \}

Nurses' professional commitment positively influences their job performance and when high, promotes positive outcomes for their patients (Donkor \& Andrews, 2011; Mrayyan \& Al-Faouri, 2008). Variables that influence professional commitment include gender, age, and monthly salary (Honyenuga \& Adzoyi, 2012) as well as educational level and years of experience (Nogueras, 2006).

In Jordan, the Health Care Accreditation Council (HCAC) provides national accreditation and is based on preventing errors, adverse events and harm to patients. Approximately 17 hospitals are accredited, and six Jordanian hospitals have received Joint Commission International (JCI) accreditation. Hospital accreditation plays an important role in improving the quality of care and safety of health care, mainly due to introducing technology, 
changing treatment patterns, opening opportunities for nurses to attend educational and training programs that enhance their knowledge and skills, and introducing standardization of care, such as incident reporting and safety policies. Although one study in Jordan studied professional commitment of nurses and its relationship to job performance( Mrayyan \& Al-Faouri, 2008), no studies have assessed professional commitment in relation to patient safety and the perceived quality care.

To investigate the professional commitment of nurses, a sample of 180 registered nurses were recruited from three hospitals in Jordan. A second aim was to investigate the relationship of nurses' professional commitment with patient safety. The expectation was that the higher the commitment, the less likely adverse events would occur.

\section{Methods}

\subsection{Setting and Sample}

The healthcare system in Jordan consists of four sectors: the Ministry of Health, Royal Medical Services, university-affiliated hospitals, and private sector hospitals. The health care services provided within these sectors are primary, secondary, and tertiary care. The 31 governmental hospitals have a total number of 4,572 beds, the 12 military hospitals have 2,428 beds, the 60 private hospitals have 3,918 beds, and the two teaching hospitals have 1,073 beds. Approximately $14.5 \%$ of the Jordanian population is admitted annually to these hospitals (Council, 2013).

Participants were selected from Jordanian accredited hospitals, as these accredited hospitals employ similar standards and policies for patient care and patient safety. The hospital settings included a governmental hospital (404 beds and 450 nurses), a private hospital (200 beds and 350 nurses), and a university affiliated hospital (526 beds and 526) nurses. To have a proportional sample, the estimated sample size was based on the workforce number and bed capacity. This resulted in recruitment of 60 registered nurses in the governmental hospital, 50 in the private hospital, and 70 in the university-affiliated hospital $(n=180)$. A convenience sample was recruited by the reserachers from the selected hospitals according to participants' availability at the time of data collection. The inclusion criteria specified registered nurses working in hospital units.

\subsection{Ethical Consideration}

Approval to conduct the study was first obtained from Ethics Committee of the primary investigators' university. The Ethics of Human Research Committee at the hospitals selected for includsion granted permission prior to nurse recruitment and data collection. Registered nurses in the potential population were asked to participate, and those who volunteered signed an informed consent form. Participants were assured that anonymity and confidentiality would be maintained throughout the study, that participation or non-participation would not affect their job standing, and that they had the right to withdrawal from the study at any time.

\subsection{Data Collection}

Nurses were asked to complete demographic information and to answer two self-administered questionnaires. The time to complete the information was approximately 10 minutes. The instruments used were the Professional Commitment Questionnaire (PCQ) and a Patient Safety Scale.

\subsubsection{Professional Commitment Questionnaire}

The questionnaire, developed by Lachman and Aranya (1986) includes four items, where each item is measured using a 7 -point scale ( $1=$ strongly disagree; $2=$ moderately disagree; $3=$ slightly disagree; $4=$ =uncertain; $5=$ slightly agree; $6=$ moderately agree; and $7=$ strongly agree). Reliability in this study resulted in Cronbach's $\alpha=0.82$.

2.3.2 Patient Safety Scale. The scale was used by Teng, Chang, and Hsu (Valiee, Peyrovi, \& Nikbakht Nasrabadi, 2014 ) in a study on patient safety in Taiwan. The instrument listed six adverse events. Nurse participants reported their perception of the frequency of these adverse events using a 9-point scale $(1=$ twice daily; $2=$ once daily; $3=$ twice weekly; $4=$ once weekly; $5=$ twice monthly; $6=$ once monthly; $7=$ twice yearly; $8=$ once yearly; and $9=$ never). Teng et al. (Teng, Chang, \& Hsu, 2009)reported Cronbach's $\alpha=.84$. Rreliability in this study resulted in Cronbach's $\alpha=0.78$.

\subsection{Data Analysis}

The study used a descriptive cross-sectional correlational research design to examine the relationship of professional commitment to patient safety and the perceived quality of patient care. Data was screened for missing data and accuracy of coding through inspecting the frequency distributions. Descriptive statistics were used to describe the sample socio-demographic characteristics and study variables. The Pearson Correlation coefficient was used to determine the relationships between professional commitment of nurses and patient safety and the 
perceived quality of patient care. An independent-sample t-test was used to identify any significant relationship between gender and professional commitment, patient safety, and the perceived quality of patient care. A one-way analysis of variance (one-way ANOVA) test was used to examine differences among commitment in the three types of hospital settings. The significance level was set at an alpha level of 0.05 . The data were analyzed using Statistical Package of Social Science (SPSS), version 22.

\section{Results}

\subsection{Characteristics of Participants}

Of the 180 questionnaires distributed to the participating nurses in the three hospitals (governmental, private, and university-affiliated), all were returned. Of these, three were incomplete and excluded from analysis. Of the 177 participants, more than half were female (53.7\%). The mean age of the study's participating nurses was 27.6 years. The mean for females ( $\mathrm{n}=95)$ was $M=3.73(S D=1.42)$ and for males $(\mathrm{n}=82)$ was $M=3.18(S D=1.71)$.

\subsection{The Level of Professional Commitment among Jordanian Registered Nurses}

The professional commitment level of Jordanian registered nurses was measured using the Professional Commitment Questionnaire (PCQ). The total score of PCQ in this study is the mean score of $\mathrm{M}=3.47(\mathrm{SD}=1.58)$ of a 7 point scale, where higher scores relate to higher commitment. According to the PCQ mean results, the highest mean score was for "loyalty to their nursing profession" $(M=4.41)$.

Table 1. Means, Standard Deviations of professional commitment among participating nurses ( $\mathrm{N}=177)$

\begin{tabular}{llll}
\hline Professional Commitment & M & SD & Level of PCQ \\
\hline PCQ & 3.47 & 1.58 & Medium \\
I feel very loyal to the nursing profession & 4.41 & 1.74 & Medium \\
For me, nursing is the best of all professions & 2.46 & 2.00 & Medium \\
I am proud to tell others that I am part of this profession & 3.24 & 2.08 & Medium \\
I really care about the fate of the nursing profession & 3.77 & 2.00 & Medium
\end{tabular}

M: mean, SD: standard deviation.

Professional commitment level: (low $=<2$; medium $=(2-5)$; high $=>5)$ out of 6 .

\subsection{The Level of Patient Safety among Jordanian Registered Nurses}

The mean for total patient safety was $\mathrm{M}=5.94(S D=1.38)$ of a 9 point scale. The mean scores for the six adverse events were as the following: Injuries due to care $(M=1.88, S D=2.09)$; Patient falls $(\mathrm{M}=1.24, \mathrm{SD}=1.55)$; Nosocomial infections $(M=1.58, S D=1.79)$; Medication administration error $(M=1.77, S D=1.79)$; Incomplete or incorrect documentations $(M=2.66, S D=2.24)$; and Delayed patient care $(M=3.16, S D=2.43)$. The lower the mean score, the higher the frequency of events perceived. The least perceived frequency related to "Delayed patient care" and the most frequently noted was "Patient falls".

\subsection{Relationship between Commitment and Patient Safety}

Pearson's product-moment correlation coefficient $(r)$ was used to examine if there was a relationship between the two continuous variables. The resulting correlation was $r=0.30 ; p<0.001$, indicating .there is a medium and positive relationship between the professional commitment of nurses and patient safety.

\subsubsection{Difference in Commitment according to Hospital Setting}

The mean PCQ score $(M=3.88, S D=1.53)$ was highest in the governmental hospital, followed by the private hospital $(M=3.51, S D=1.68)$ and university-affiliated teaching hospitals $(M=3.05, S D=1.44)$. A one-way ANOVA) was used to determine and compare the variances among different hospital types and the level of the professional commitment of nurses. A statistically significant difference was found $(F(2,174)=4.33, p=0.01)$.

The statistical difference in professional commitment of nurses among different hospital settings was determined using post-hoc comparisons based on Tukey's HSD test. The mean for the governmental hospital $(M=3.88$, $S D=1.53$ ) was significantly higher than the mean of the private and the university- affiliated hospital.

\subsubsection{Relationship between Demographical Variables and Commitment}

An independent-sample t-test conducted to assess the relationship between professional commitment of nurses and 
gender showed a significant negative relationship $t(158=-2.33 ; \mathrm{p}=.02)$ as shown in Table 2. The Pearson correlation coefficient used to examine the relationship between the professional commitment of nurses and their age and years of experience in nursing showed no significant relationships. A low and negative relationship was found in relation of years of experience in the current hospital $(r=-0.193 ; p=0.01)$. A One- way ANOVA used to examine the relationship among the level the professional commitment of nurses resulted in a statistically significant relationship between professional commitment and current hospital sector $(F=4.334, p=0.01)$. Furthermore, a statistically significant relationship was found between the monthly salary of nurses and professional commitment $(F=12.327, p=0.000)$.

Table 2. Summary of results related to professional commitment of nurses and selected demographic variables $(\mathrm{N}=177)$

\begin{tabular}{|c|c|c|c|c|c|c|}
\hline Variables & $\mathrm{N}$ & $\mathrm{M}(\mathrm{SD})$ & $t$ & $P$ & F-test & $r$ \\
\hline Gender & & & -2.33 & $0.02 *$ & & \\
\hline Female & 95 & $3.73(1.42)$ & & & & \\
\hline Male & 82 & $3.18(1.71)$ & & & & \\
\hline Age & & 27 & & 0.51 & & $-.049 * *$ \\
\hline Experience in years & & 5.1 & & 0.54 & & $-.045^{* *}$ \\
\hline Current yrs. in hospital & & 3.6 & & 0.01 & & $-.193 * *$ \\
\hline Marital status & & & & 0.67 & 0.392 & \\
\hline Single & 90 & $3.37(1.73)$ & & & & \\
\hline Married & 83 & $3.58(1.44)$ & & & & \\
\hline Other & 04 & $3.63(0.82)$ & & & & \\
\hline Educational level & & & & 0.27 & 1.303 & \\
\hline Diploma & 09 & $4.19(1.40)$ & & & & \\
\hline Bachelor & 156 & $3.46(1.59)$ & & & & \\
\hline Master's & 12 & $3.08(1.50)$ & & & & \\
\hline Hospital sector & & & & $0.01^{*}$ & 4.334 & \\
\hline Governmental & 60 & $3.88(1.53)$ & & & & \\
\hline University & 62 & $3.05(1.44)$ & & & & \\
\hline Private & 55 & $3.51(1.68)$ & & & & \\
\hline Monthly salary & & & & $0.000^{*}$ & 12.32 & \\
\hline Between (300-500)JD & 107 & $3.91(1.53)$ & & & & \\
\hline Between (501-1000)JD & 69 & $2.82(1.42)$ & & & & \\
\hline More than 1000JD & 01 & 1.25 & & & & \\
\hline
\end{tabular}

M, mean; SD, standard deviation; ${ }^{*} p$ value significant at $<0.05 ; * *$ Correlation is significant at the 0.01 level (2-tailed); F value, using one-way ANOVA; $t$ value, using independent $t$-test analysis.

\subsection{Relationship of Demographic Variables and Patient Safety}

An independent-sample t-test used to assess the relationship between patient safety and gender showed no statistically significant relationship. Likewise, the Pearson correlation coefficient used to examine the relationship between patient safety and nurse's age, years of experience in nursing, and years of nursing experience in their current hospital showed no statistically significant. A one-way ANOVA showed a statistically significant relationship between patient safety and educational level $(F=3.306, p=0.03)$, whereas no statistically significant relationship related to marital status, current hospital, and monthly salary. The results are presented in Table 3 . 
Table 3. Summary of results related to patient safety and different demographical variables among nurses' participants $(\mathrm{N}=177)$

\begin{tabular}{|c|c|c|c|c|c|c|c|}
\hline \multicolumn{2}{|l|}{ Variables } & $\mathrm{N}$ & $\mathrm{M}(\mathrm{SD})$ & $t$ & $P$ & $F$ & $r$ \\
\hline \multicolumn{2}{|l|}{ Gender } & & & -0.337 & 0.73 & & \\
\hline \multicolumn{2}{|l|}{ Female } & 95 & $5.98(1.39)$ & & & & \\
\hline \multicolumn{2}{|l|}{ Male } & 82 & $5.91(1.37)$ & & & & \\
\hline \multicolumn{2}{|l|}{ Age } & & & & 0.17 & & 0.103 \\
\hline \multicolumn{2}{|l|}{ Experience in years } & & & & 0.37 & & 0.067 \\
\hline \multicolumn{2}{|c|}{ Current hospital experience } & & & & 0.34 & & -0.071 \\
\hline \multicolumn{2}{|l|}{ Marital status } & & & & 0.54 & 0.619 & \\
\hline \multicolumn{2}{|l|}{ Single } & 90 & $5.93(1.39)$ & & & & \\
\hline \multicolumn{2}{|l|}{ Married } & 83 & $5.93(1.37)$ & & & & \\
\hline \multicolumn{2}{|l|}{ Other } & 04 & $6.71(1.20)$ & & & & \\
\hline \multicolumn{2}{|l|}{ Educational level } & & & & $0.03 *$ & 3.306 & \\
\hline \multicolumn{2}{|l|}{ Diploma } & 09 & $6.48(0.93)$ & & & & \\
\hline \multicolumn{2}{|l|}{ Bachelor } & 156 & $5.94(1.35)$ & & & & \\
\hline \multicolumn{2}{|l|}{ Master } & 12 & $5.06(1.77)$ & & 0.06 & 2.952 & \\
\hline \multicolumn{8}{|l|}{ Hospital sector } \\
\hline \multicolumn{2}{|l|}{ Governmental } & 60 & $3.88(1.53)$ & & & & \\
\hline \multicolumn{2}{|l|}{ University } & 62 & $3.05(1.44)$ & & & & \\
\hline \multicolumn{2}{|l|}{ Private } & 55 & $3.51(1.68)$ & & 0.08 & 2.55 & \\
\hline \multicolumn{8}{|l|}{ Monthly salary } \\
\hline Between (300-500)JD & 107 & 107 & $6.13(1.28)$ & & & & \\
\hline Between (501-1000)JD & 69 & 69 & $5.66(1.50)$ & & & & \\
\hline More than $1000 \mathrm{JD}$ & 1 & 01 & $6.50-$ & & & & \\
\hline
\end{tabular}

$\mathrm{M}$, mean; SD, standard deviation; * $p$ value significant at $<0.05$; ${ }^{* *}$ Correlation is significant at the 0.01 level (2-tailed); F value, using one-way ANOVA; $\mathrm{t}$ value, using independent $\mathrm{t}$-test analysis.

\section{Discussion}

\subsection{Professional Commitment}

The mean age of the nurses in the sample was relatively young (27.6 years) and almost split in gender, with $53.7 \%$ being female. However, these findings are consistent with the mean age and gender division of the nursing workforce in Jordan $(\mathrm{JNC}, 2013)$. The total mean score $(\mathrm{m}=3.48)$ for professional commitment is mid-point of a 07 point scale, constituting a medium level of commitment. This finding is similar with results of a study by ( Mrayyan \& Al - Faouri, 2008) that examined the level of professional commitment among Jordanian nurses. However, the level of commitment found in the current study appears to be lower than those seen in reviewed literature. Seruya and Hinojosa(Seruya \& Hinojosa, 2010) attributed a higher level of commitment due to the nurses' practice setting. Honyenuga and Adzoyi (Honyenuga \& Adzoyi, 2012) in a study conducted in Ghana considered that financial gain and love for nursing as a profession contributed to a high level of commitment.

According to the PCQ mean results, nurses in the study felt loyal to their nursing profession $(M=4.41)$, while they were moderately committed in reference to the remaining items. This result may be due to lack of proper preparation or experience of newly graduated nurses in entry in the nursing profession. If this is so, a solution for the nurses in transition from training in schools to practice in hospitals may be to clarify the nursing role and job description (Grealish \& Smale, 2011). Also, proper preparation through educational and training courses might enhance commitment (Coogle, Parham, Jablonski, \& Rachel, 2007).

The finding of moderate commitment $\mathrm{M}=3.47$ ( $\mathrm{SD}=1.58)$, may be due to factors such as the need for a better salary 
and opportunity for advancement. However, most hospital nurses get financial incentives. The Jordanian Nursing Council provides educational and training programs, such as cardiac life support, communication skills, and leadership for change. In addition, Jordanian hospitals present opportunities for their nurses to attend national and international workshops or conferences.

\subsection{Professional Commitment in Terms of Hospital Setting}

Seruya and Hinojosa(Seruya \& Hinojosa, 2010) suggested that the nurses' practice setting has an effect on their professional commitment. In examining the differences in professional commitment of nurses according to hospital settings, the results of this study showed that there was a statistically significant difference in professional commitment of nurses according to hospital settings (governmental, university-affiliated, and private hospitals). Registered nurses working in the governmental hospital were more committed to the nursing profession than those in private and university-affiliated hospitals. This result is inconsistent with the finding of ( Mrayyan \& Al Faouri, 2008), who reported that Jordanian nurses working in the private hospitals were more committed than nurses working in university and governmental hospitals. In addition, Breau and Réaume (Breau \& Réaume, 2014) found that nurses significantly differed in their job satisfaction in terms of their work environment: nurses who experienced better work environment had higher job satisfaction than who had an inferior work environment.

In Jordan, the moderate professional commitment of nurses of governmental hospital (3.88) might be linked to job security. The government hospital provides benefits of transportation and housing facilities for female nurses. Most of the nurses working in the private hospital had retired from military or governmental hospitals and, consequently were older than those in university and governmental hospitals. However, salaries are lower in the government hospitals than in the private hospitals, thereby exerting a negative perception.

On the other hand, nurses in private and university hospitals may feel more stress, as nursing errors threatens advancement in their profession within the hospital. There also tends to be more shifting of nurses from their usual ward to those that are short-handed. Furthermore, nurses in private and university-affiliated hospitals tend to migrate to other counties where they can receive higher wages.

\subsection{Demographic Variables and Professional Commitment}

Honyenuga and Adzoyi (Honyenuga \& Adzoyi, 2012) found a significant relationship between the professional commitment of nurses and their gender, financial gain, and hospital setting. Findings of the current study indicated that female nurses are more willing to and have the ability to work various nursing services than the male nurses. This may be due to the general Jordanian culture, where nursing is traditionally considered to be a profession for females. However, monthly salary was the strongest predictor influencing professional commitment. The opportunity for advancement and higher pay for nurses in Jordan is a positive influence. In terms of careers for women, nursing is considered a high priority profession. This result is consistent with a finding by Honyenuga and Adzoyi(Honyenuga \& Adzoyi, 2012) that reported two third of nurses were committed to their profession due to financial causes. Years of experience in their current hospital significantly negatively correlated with commitment, which indicates that as years of experience increased, the nurses' commitment to the nursing profession decreased. Again, this might also be related to financial aspects. If nurses perceive that wages do not keep up with their experience and skills, their commitment might lessen.

This study showed no significant correlation between years of experience in nursing, age, marital status, or educational level to the level of the professional commitment. This result is congruent with (LeDuc \& Kotzer, 2009), who found no significant relationship in relation to years of expertise. Also, Teng et al. (Teng et al., 2009) found no relationship between the professional commitment and marital status. The current study finding of the relationship with higher education differs from that of the study of Nogueras (Nogueras, 2006), who found that nurses who had high educational degrees and more years of experience in nursing were more committed to the nursing profession. Likewise, Mrayyan and Al Faouri (Majd T Mrayyan \& Al - Faouri, 2008) found a significant relationship between age, educational level, marital status, and years of nursing experience to the professional commitment of nurses. Honyenuga and Adzoyi (Honyenuga \& Adzoyi, 2012) reported that there is a significant relationship between the professional commitment of nurses and their age.

\subsection{Patient Safety}

The mean score $(M=5.94)$ for patient safety was higher than mid-point on a 9 point scale. This suggests that nurses moderately perceived and countered adverse patient safety issues. This differs from results found by Feng et al. (Feng, Acord, Cheng, Zeng, \& Song, 2011) who examined the level of patient safety culture among staff nurses and nurse managers. The authors noted that a staff nurses produced lower scores that nurse managers. The result of the current study could be due to the fact that the hospitals in the study setting were accredited; patient safety 
levels had been improved hospitals in these hospitals and therefore, overall, the nurses reported fewer adverse events.

Kagan and Barnoy and Nygren et al (Kagan \& Barnoy, 2013; Nygren et al., 2013),have noted that many factors (e.g. incident reporting, risk management, patient education, patient-nurse interaction, and compliance to patient safety law) influenced patient safety. Accordingly, nurses were more likely to be in compliance with patient safety standards and policies, which are considered a requirement for hospital accreditation. In addition, continuous managerial support through frequent feedback and encouragement may enhance nurse care and attention to patients, which in turn, enhances the level of patient safety.

This study found a medium positive relationship between professional commitment and patient safety. One interpretation is that nurses who are committed to the nursing profession were able to maintain and enhance patient safety better than those who are not professionally committed. This result is similar with the Teng et al. (Teng et al., 2009) finding of a significant positive relationship between the professional commitment of nurses and patient safety. Armellino, Griffen, and Fitzpatrick (Armellino, QUINN GRIFFIN, \& Fitzpatrick, 2010) reported a significant correlation between structural empowerment and a patient safety culture. Also, Yang, Liu, Huang, and Zhu (Yang, Liu, Huang, \& Zhu, 2013) reported that the correlation between nurse empowerment and patient safety was mediated by professional and organizational commitment.

Another study (Boltz, Capezuti, Wagner, Rosenberg, \& Secic, 2013) reported a significant inverse relationship between nurse certification in any specialty and patient fall rate and noted this might be due to the heavy work-load. This may be especially true in accredited hospitals that provide lectures and risk management courses, where attendance adds to the workload burden. However, the process of providing continuous direct and indirect observation, establishing evidenced-based policies, and ensuring incident reporting may provide hospital nurses with enough knowledge and experience that they can relate to a proper and safe work environment. Additionally, nurses who are committed to the nursing profession are more likely to comply with compliance accreditation requirements and hospital directives.

\subsection{Patient Safety According to Hospital Setting}

The finding of no statistically significant difference among the three hospital settings (governmental, university-affiliated, and private hospitals) suggests that nurse perception of patient safety was essentially similar. Because all three hospitals are accredited and adhere to the same patient safety standards, nurses adhere to policies aimed to prevent or decrease adverse events and, by necessity, attend to more complete documentation and incident reporting. Kagan and Barnoy (Kagan \& Barnoy, 2013) found that reporting errors was significantly influenced by a patient safety culture. In addition, Feng, Acord, Cheng, Zeng, and Song (Feng et al., 2011) note that a patient safety culture is influenced by nurse compliance to national goals that focus on patient safety. Steelman, Graling, and Perkhounkova (Steelman, Graling, \& Perkhounkova, 2013) suggested that communicating safety related information to hospital nurses improves their nursing practices.

\subsection{Demographic Variables and Patient Safety}

The variable showing a significant relationship with patient safety was education. In Jordan, registered nurses often hold higher degrees because a nurse with a Bachelor degree is eligible for higher wages than a nurse without a college degree. Awareness of safety regulations, especially in regard to documenting patient care and following doctor's directives, is more likely to occur. Robson et al. (Robson et al., 2013) note it is essential to increase nurse awareness regarding patient safety. This is supported by the results of Valiee et al. (Valiee et al., 2014), who reported causes that lead to nursing errors include nurse's lack of knowledge, or awareness of consequences of events, and lack of evidence-based practice.

\section{Implication for Clinical Practice}

In Jordan, no study was found that investigated the relationship among the professional commitment of nurses and patient safety. The results of this study will provide baseline information to guide nurse educators and nurse managers to develop hospital policies and incentive-based strategies designed to enhance and maintain professional commitment. Nurse Managers have an opportunity to enhance the professional commitment of their staff nurses through actions such as promoting a better work environment, encouraging teamwork, and providing instruction and feedback on preventing adverse clinical events. These activities, in turn, are expected to increase staff nurse awareness of patient safety issues. The working hypothesis is a committed nurse works to reduce adverse effects, which in turn, enhances patient safety. 


\section{Conclusion}

This study suggested that professional commitment and issues related to patient safety among Jordanian Registered Nurses correlate significantly. However, the level of commitment of the Registered Nurses measured mid-point on the scale, indicating a moderate yet positive level of professional commitment. The study participants, which included an almost equal number of male and female nurses from three differing types of hospitals (government, private, and university-affiliated) perceived a range of adverse events, with delay in patient care being the least frequently seen adverse event, in their work setting. Nurses' commitment was influenced by gender, number of years of nursing experience in their current hospital, current hospital sector and monthly salary. The total mean scores revealed a significant difference among hospitals in terms of the professional commitment of nurses; the highest mean was found among nurses working in governmental hospitals.

\section{Funding}

This research received no specific grant from any funding agency in the public, commercial, or not-for-profit sectors.

\section{Competing Interests Statement}

No conflict of interest has been declared by the authors.

\section{References}

Armellino, D., Quinn Griffin, M. T., \& Fitzpatrick, J. J. (2010). Structural empowerment and patient safety culture among registered nurses working in adult critical care units. $J$ Nurs Manag, 18(7), 796-803. https://doi.org/10.1111/j.1365-2834.2010.01130.x

Boltz, M., Capezuti, E., Wagner, L., Rosenberg, M. C., \& Secic, M. (2013). Patient safety in medical-surgical units: can nurse certification make a difference?. Medsurg Nursing, 22(1), 26-32, 37. PMID:23469496.

Breau, M., \& Réaume, A. (2014). The relationship between empowerment and work environment on job satisfaction, intent to leave, and quality of care among ICU nurses. Dynamics, 25(3), 16-24.

Coogle, C. L., Parham, I. A., Jablonski, R., \& Rachel, J. A. (2007). The value of geriatric care enhancement training for direct service workers. Gerontology \& Geriatrics Education, 28(2), 109-31. https://doi.org/10.1300/J021v28n02_08

Jordanian Nursing Council. (2013). Current and Projected Nursing Manpower in Jordan (JNC).

Donkor, N. T., \& Andrews, L. D. (2011). Ethics, culture and nursing practice in ghana. International Nursing Review, 58(1), 109. https://doi.org/10.1111/j.1466-7657.2010.00852.x

Feng, X. Q., Acord, L., Cheng, Y. J., Zeng, J. H., \& Song, J. P. (2011). The relationship between management safety commitment and patient safety culture. International Nursing Review, 58(2), 249. https://doi.org/10.1111/j.1466-7657.2011.00891.x

Gaal, S., Van, L. E., Wolters, R., Wetzels, R., Verstappen, W., \& Wensing, M. (2010). Patient safety in primary care has many aspects: an interview study in primary care doctors and nurses. Journal of Evaluation in Clinical Practice, 16(3), 639. https://doi.org/10.1111/j.1365-2753.2010.01448.x

Grealish, L., \& Smale, L. A. (2011). Theory before practice: implicit assumptions about clinical nursing education in australia as revealed through a shared critical reflection. Contemporary Nurse, 39(1), 51-64. https://doi.org/10.5172/conu.2011.39.1.51

Honyenuga, B., \& Adzoyi, P. (2012). Professional commitment of nurses in ghana: the case of the volta region. Asia Pacific Journal of Business and Management, 3(1), 12-27.

Kagan, I., \& Barnoy, S. (2013). Organizational safety culture and medical error reporting by israeli nurses. Journal of Nursing Scholarship An Official Publication of Sigma Theta Tau International Honor Society of Nursing, 45(3), 273-80. https://doi.org/10.1111/jnu.12026

Leduc, K., \& Kotzer, A. M. (2009). Bridging the gap: a comparison of the professional nursing values of students, new graduates, and seasoned professionals. Nursing Education Perspectives, 30(5), 279.

Mrayyan, M. T. (2012). Reported incidence, causes, and reporting of medication errors in teaching hospitals in jordan: a comparative study. Contemporary Nurse, 41(2), 216-232. https://doi.org/10.5172/conu.2012.41.2.216 
Mrayyan, M., \& Al-Faouri, I. (2008). Career commitment and job performance of jordanian nurses. Nursing Forum, 43(1), 24-37. https://doi.org/10.1111/j.1744-6198.2008.00092.x

Nogueras, D. J. (2006). Occupational commitment, education, and experience as a predictor of intent to leave the nursing profession. Nursing Economic, 24(2), 86.

Nygren, M., et al. (2013). Factors influencing patient safety in Sweden: perceptions of patient safety officers in the county councils. BMC health services research, 13(1), 52. https://doi.org/10.1186/1472-6963-13-52

Robson, W., Clark, D., Pinnock, D., White, N., \& Baxendale, B. (2013). Teaching patient safety and human factors in undergraduate nursing curricula in england: a pilot survey. British Journal of Nursing, 22(17), 1001. https://doi.org/10.12968/bjon.2013.22.17.1001

Seruya, F. M., \& Hinojosa, J. (2010). Professional and organizational commitment in paediatric occupational therapists: the influence of practice setting. Occupational Therapy International, 17(3), 125. https://doi.org/10.1002/oti.293

Smits, M., Wagner, C., Spreeuwenberg, P., Timmermans, D. R., Van, d. W. G., \& Groenewegen, P. P. (2012). The role of patient safety culture in the causation of unintended events in hospitals. Journal of Clinical Nursing, 21(23-24), 3392-3401. https://doi.org/10.1111/j.1365-2702.2012.04261.x

Steelman, V. M., Graling, P. R., \& Perkhounkova, Y. (2013). Priority patient safety issues identified by perioperative nurses. Aorn Journal, 97(4), 402. https://doi.org/10.1016/j.aorn.2012.06.016

Teng, C. I., Chang, S. S., \& Hsu, K. H. (2009). Emotional stability of nurses: impact on patient safety. Journal of Advanced Nursing, 65(10), 2088-96. https://doi.org/10.1111/j.1365-2648.2009.05072.x

Valiee, S., Peyrovi, H., \& Nasrabadi, A. N. (2013). Critical care nurses' perception of nursing error and its causes: a qualitative study. Contemporary Nurse, 46(2), 206-213. https://doi.org/10.5172/conu.2014.46.2.206

Yang, J. H., Liu, Y., Huang, C. H., \& Zhu, L. F. (2013). Impact of empowerment on professional practice environments and organizational commitment among nurses: a structural equation approach. International Journal of Nursing Practice, 19(S1), 44-55. https://doi.org/10.1111/ijn.12016

\section{Copyrights}

Copyright for this article is retained by the author(s), with first publication rights granted to the journal.

This is an open-access article distributed under the terms and conditions of the Creative Commons Attribution license (http://creativecommons.org/licenses/by/4.0/). 